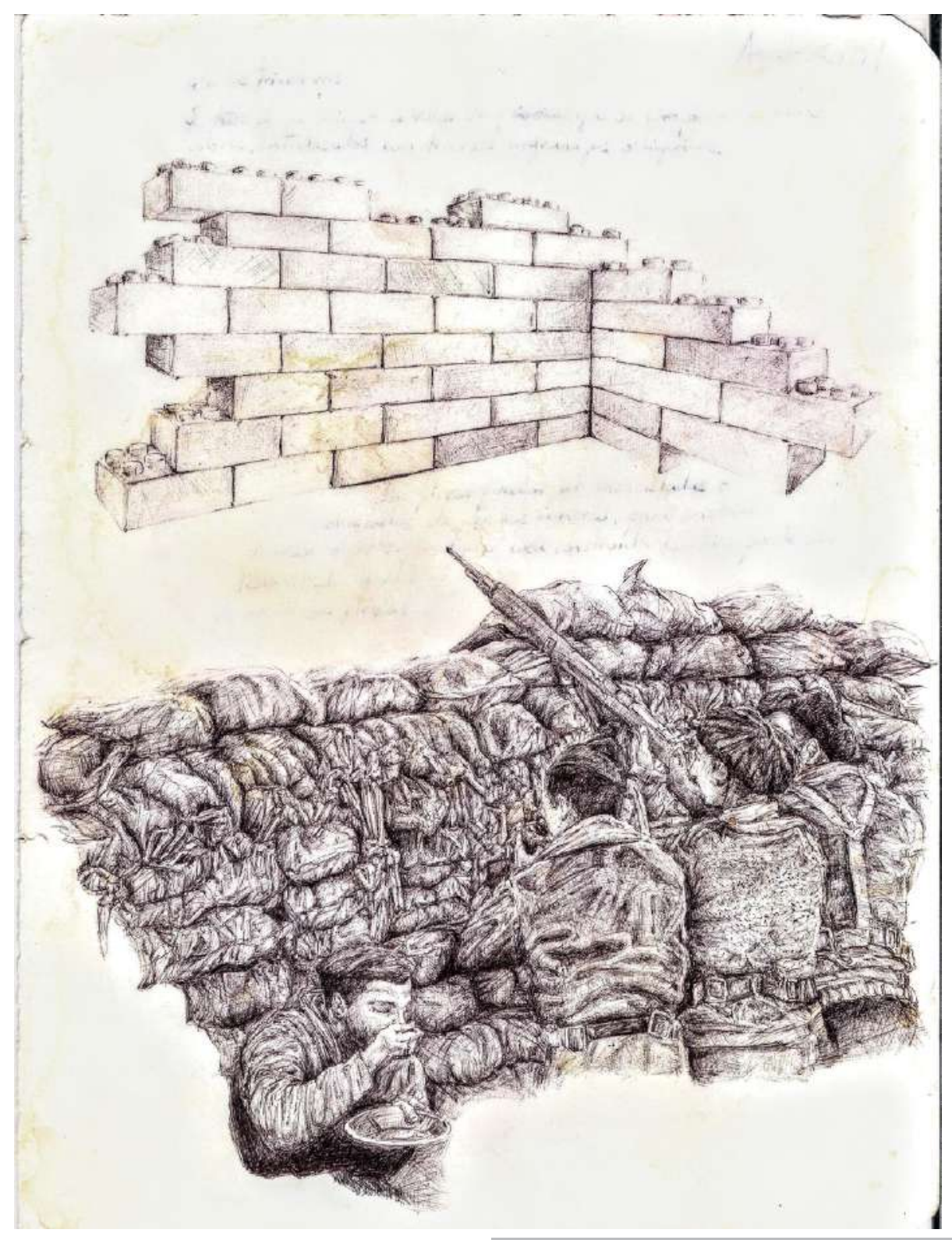

Sebastián Zea Quintero

Sin título

De la serie Del juego y el hombre Lápiz sobre papel

2017

Medellín 


\title{
La disputa por el territorio en el cambio en la Política de Ordenamiento Territorial de Manizales (2003-2017). Un estudio a partir del marco de análisis y desarrollo institucional*
}

\author{
Manuela Carmona Bedoya (Colombia) ${ }^{* *}$
}

\section{Resumen}

Se realiza un análisis de la política de Ordenamiento Territorial en la ciudad de Manizales a partir de 2003, a raíz del cambio de la norma para el uso del suelo en La Aurora, área colindante a la Reserva Forestal de Río Blanco. Mediante un método de investigación cualitativa y usando el marco de análisis y desarrollo institucional (ADI) se establece que si bien las reglas constitucionales determinan la formulación de los planes de ordenamiento territorial (POT) estas desencadenan un espacio de interacción, en el que los actores locales desarrollan estrategias para modificar dichas reglas. Se concluye que en este proceso los participantes desarrollan un aprendizaje incremental en el que logran modificar las reglas, unas veces desde una perspectiva de corto plazo y en favor de intereses particulares o reducidos, y otras veces con una visión de más largo plazo con arreglo a valores y en beneficio de la naturaleza y de las generaciones futuras. En cada caso la capacidad de la acción colectiva es determinante.

\section{Palabras clave}

Políticas Públicas; Política Ambiental; Política Territorial; Política Local; Acción Colectiva; Análisis y Desarrollo Institucional (ADI).

Fecha de recepción: octubre de 2019

- $\quad$ Fecha de aprobación: abril de 2020

\section{Cómo citar este artículo}

Carmona Bedoya, Manuela. (2020). La disputa por el territorio en el cambio en la Política de Ordenamiento Territorial de Manizales (2003-2017). Un estudio a partir del marco de análisis y desarrollo institucional. Estudios Políticos (Universidad de Antioquia), 58. pp. 168-192. DOI: 10.17533/udea.espo.n58a08

\footnotetext{
* Este artículo se deriva del proceso de investigación de la tesis Resignificar el territorio ¿expansión o conservación de las fuentes de agua en Manizales? Análisis de la política de ordenamiento territorial entre los años 2001 y 2017, para optar al título de maestría de Investigación en Políticas Públicas, Facultad Latinoamericana de Ciencias Sociales (Flacso)-Ecuador.

** Abogada. Administradora Pública. Correo electrónico: manucarmona610@gmail.com - Orcid: 00000002-2701-220X - Google Scholar: https://scholar.google.es/citations?hl=es\&user=3Et2pWwAAAAJ
} 


\title{
The Dispute over the Territory in the Change of the Land Use Policy in Manizales (2003-2017). A Study Based on the Institutional Analysis and Development Framework
}

\begin{abstract}
As a result of the change in the norm for the land use in La Aurora, an adjacent area to the Río Blanco Forest Reserve, this study analyses the Territorial Planning Policy for the city of Manizales since 2003. Through a qualitative research methodology and based on the Institutional Analysis and Development Framework (IAD), the article establishes that even if constitutional rules determine the formulation of Land Use Plans (POT), these rules present a gap, in which local actors develop strategies to modify them. As a conclusion, the study states that, in this process, the participants develop incremental learning in which they manage to modify the rules, sometimes from a short-term perspective and favoring particular or reduced interests, and sometimes with a longer-term vision according to values and in benefit of nature and future generations. In each case, the capacity for collective action is decisive.
\end{abstract}

\section{Keywords}

Public Politics; Environmental Politics; Territorial Politics; Local Politics; Collective Action; Institutional Analysis and Development. 


\section{Introducción}

En la década de 1970 los países del Sur comenzaron a insistir en la protección al medio ambiente como parte de las políticas que se implementaban para promover el desarrollo (Estenssoro, 2015). Se exigía una relación casi simbiótica entre la protección a los recursos naturales y los asentamientos humanos. Surgiendo con ello la idea del ecodesarrollo. América Latina adopta este enfoque en la década de 1980, buscando un desarrollo sostenible en la ordenación del territorio que integrara objetivos ambientales en función de la planificación físico-espacial (Massiris, 2002). En 1992, con la Declaración de Río sobre el Medio Ambiente y Desarrollo, se establece una agenda internacional para crear una normativa fuerte que protegiera el medio ambiente y a la vez promoviera la participación ciudadana en la toma de decisiones públicas.

En 1991 Colombia promulgó la primera Constitución Política considerada a su vez como ecológica o ambiental, en ella se consagra de forma explícita un apartado de derechos colectivos y del ambiente, y se sobrepone el medio ambiente a los planos económico e individual, haciendo que las actuaciones públicas se sujetaran al interés general, a la protección de la dignidad humana

y a la primacía de los valores comunes (Corte Constitucional, Sentencia C-339 de 2002).

Este principio general de la primacía del bien común sobre el particular impactó en todo el ordenamiento territorial del país. Con la Ley 388 de 1997 se exige a los municipios adecuar sus territorios de acuerdo a los recursos naturales que posean, respetando y evitando acciones lesivas a estos bienes comunes. Se reconoce de esta forma la diversidad ambiental que existe en el país.

La investigación corresponde al estudio de caso extremo de la ciudad de Manizales, departamento de Caldas entre 2003, con el cambio en el POT que convierte en área de expansión el terreno aledaño a la Reserva Forestal de Río Blanco, hasta 2017, cuando el POT es modificado nuevamente. Allí radica el problema de política pública asociable a la gobernanza del agua, en torno al uso del suelo referente a recursos no renovables que generan servicios ecosistémicos de regulación hídrica y climática para la población de toda una ciudad. Es un aporte práctico al uso del modelo del análisis del desarrollo institucional (ADI), con aplicación en el uso de recursos 
La disputa por el territorio en el cambio en la Política de Ordenamiento Territorial...

hídricos y reservas forestales en Latinoamérica, centrado en Colombia. Con la utilización del ADI, se evidencia cómo este caso puede engrosar la teoría generada por Elinor Ostrom donde el análisis de un particular permite comprender cómo se genera acción colectiva para mantener la protección de un RUC que es manejado por la autoridad local, y la relación entre actores que modifican las reglas e instituciones de los Recursos de Uso Común, en este caso, el agua.

En el departamento de Caldas se encuentran bajo protección jurídica, además de la Reserva Forestal Protectora de Río Blanco y Quebrada Olivares, «el Parque Nacional Natural los Nevados, Reserva Forestal de La CHEC, Reserva Forestal Torre Iv y el Parque Nacional Natural Selva de Florencia» (Corpocaldas, 2010, p. 13). Para el municipio de Manizales, Río Blanco representa la provisión de $35 \%$ del agua potable a la ciudad (p. 67). Esta reserva es un bosque de niebla, un ecosistema único y estratégico, de un gran valor e influencia para el bienestar humano por su esencial papel como regulador del ciclo hídrico y del mantenimiento de las fuentes de agua, y porque al ser sumidero para el secuestro de carbono, fuente de estabilidad climática, provee especies útiles y promisorias, o puede proporcionar ingresos alternativos para las poblaciones locales (Armenteras, Cadena y Moreno, 2007, p. 16).

La importancia de los bosques de niebla evidencia la necesidad de valorar los beneficios ecosistémicos que presta Río Blanco a Manizales, no solo en la provisión de agua potable, sino también en la conservación de especies endémicas, en servir de corredor biológico para el paso de especies de fauna, la generación de un microclima que beneficia a la ciudad, el control del ciclo hídrico, entre otros. Es necesaria la protección de la reserva por parte de la ciudad mediante una organización territorial que respete y sostenga la vida, no solo en la reserva, sino también en su zona aledaña.

La gobernanza permite la interacción y el intercambio de conocimientos, ideas, recursos y normas de actores que quieren formular y alcanzar objetivos comunes, donde la comunidad toma fuerza en su nivel de participación frente al poder del Estado y del mercado (Zurbriggen, 2011). La gobernanza comunitaria evidencia la capacidad de los actores en el manejo de los problemas locales porque «cuenta con información privada dispersa que algunas veces no está disponible para los estados, empleadores, bancos y 
otras organizaciones formales» (Bowles, 2004, p. 19), donde interactúan reglas que determinan las relaciones.

Por medio del marco de ADI se estudian los planes de ordenamiento territorial (POT) de Manizales desde 2003 hasta 2017 para comprender cómo la utilización de la Ley 388 de 1997 —entendida como la regla constitucional - impulsa los intereses de los actores. Estos intereses son de dos tipos: el primero promueve la construcción de una urbanización en el terreno aledaño y colindante a la reserva, denominado La Aurora; el segundo pretende mantener como rural este sector para beneficio de las generaciones futuras y de los demás seres vivos qu sostienen el ciclo hídrico, el cual inicia con la captación del agua lluvia.

Este análisis del cambio en los POT de la ciudad de Manizales debe entenderse como una comprensión de política pública según la propuesta de André-Noel Roth (2010; 2018), ya que combina la acción de autoridades públicas, principalmente de la Alcaldía de Manizales y la Corporación Autónoma Regional de Caldas (Corpocaldas), enfocadas en resolver insatisfacciones ciudadanas evidenciadas en la necesidad de promover el acceso a la vivienda y, posteriormente, en la protección ambiental.

La pregunta que guía este artículo es: ¿cómo utilizan los participantes la regla constitucional para establecer el cambio en el ordenamiento territorial de la ciudad de Manizales en el periodo 2003-2017 en torno a la Reserva Forestal de Río Blanco? La hipótesis, basada teóricamente en el ADI, afirma que la existencia de una jerarquía normativa donde la regla constitucional determina a la de elección colectiva y a las demás en uso establece el alcance de las acciones que utilicen los actores para influenciar en el cambio sobre el POT.

\section{Marco teórico y conceptual}

\subsection{Recursos de uso común}

Garret Hardin (1968) expuso el problema de la gestión de los recursos comunes, que en la mayoría de los casos desembocaba en una tragedia. Pone como ejemplo la existencia de un campo compartido por ganaderos, quienes utilizan de forma individual e irresponsable el pastizal que comparten 
La disputa por el territorio en el cambio en la Política de Ordenamiento Territorial...

para aumentar sus beneficios, disminuyendo la utilización para los demás y generando la degradación a futuro del entorno. La solución a esta toma de decisiones individuales sería el establecimiento de un mecanismo de control externo, el cual podría realizarse por parte del Estado o del mercado, siempre y cuando se mitigara el abuso sobre los recursos que tienen en común varios individuos.

Elinor Ostrom (2015a) plantea que la gestión comunitaria es una tercera vía en la gestión de los recursos de uso común (RUC). Los RUC se caracterizan por cumplir dos propiedades: la dificultad de excluir beneficiarios es alta y la sustracción del recurso por parte de un participante disminuye su uso para los demás. Es decir, una persona no puede ser excluida del uso del agua en un sistema de riego compartido y en su parcela el recurso hídrico utilizado ya no podrá existir para otro usuario. A su vez, los RUC se dividen en sistemas de recursos caracterizados por ser las «variables de acervo capaces, en circunstancias favorables, de producir una cantidad máxima de un flujo variable de unidades del RUC sin perjudicar el acervo o el sistema de recursos», y en unidades del recurso, «entendidas como aquello que usan o se apropian los individuos del sistema de recursos» (p. 77).

Es necesario establecer que si bien Ostrom (2015b) plantea la gestión comunitaria como la adecuada para manejar a los recursos de uso común, también enfatiza en la acción colectiva como un elemento clave para comprender que la ciudadanía puede ser parte activa en la protección de su entorno. De esta forma, la gestión del RUC puede realizarla el Estado o el mercado, pero existe una fuerte presencia de la sociedad en el control y la vigilancia de esta tarea.

La acción colectiva es el resultado del aprendizaje estratégico de los participantes que se encuentran insertos en una arena de acción. Estas personas generan acuerdos basados en la confianza y la comunicación que desarrolla un ejercicio de ensayo-error en las relaciones para proponer unas reglas de uso estables en el tiempo que les permitan mantener el statu quo por medio de acuerdos institucionales efectivos (Ostrom, 2015a) del RUC que vigilan. Para Ostrom (2015b), los RUC hacen parte de la caracterización biofísica del entorno que afecta a la arena de acción. Con ella se determina la gestión, el aprovechamiento y la utilización del recurso por parte de los participantes, generando un condicionamiento del tipo de reglas que influyen sobre la situación de acción. 


\subsection{El marco de análisis y desarrollo institucional (ADI)}

Dentro del neoinstitucionalismo de la elección racional surge la nueva economía institucional (NEI), la cual pretende comprender cómo los incentivos determinan el funcionamiento de las economías a partir de las elecciones individuales. Elinor Ostrom, con su propuesta de la «gobernanza de los bienes comunes» (Caballero, 2011, p. 14), se inscribe en esta corriente.

En el ADI las reglas son sumamente relevantes, determinan la forma en que se toman las decisiones, las acciones permitidas o prohibidas, los procedimientos, la información que se utiliza, entre otros (Ostrom, 2015a, p. 109). Lo más importante es que permiten establecer un arreglo institucional que genere mayores beneficios a los participantes, según las estrategias que utilicen. Las reglas son una institución poderosa en la toma de decisiones. Se entiende al ADI como un marco analítico que permite evidenciar el cambio en las instituciones a lo largo del tiempo, en una arena de acción determinada que motiva a los participantes a movilizarse mediante acción colectiva para mantener o modificar el statu quo que una regla le ha impuesto, estableciendo nuevas relaciones con el entorno.

[174] Podría pensarse que el ADI se enmarcaría en una epistemología positivista bajo el análisis de la elección racional; sin embargo, se reconoce que casa caso es único, permitiendo comprender que la especificidad en cada situación impide la generalización. Esto evidencia una adaptación del modelo a las preferencias epistemológicas del investigador, al presentar un análisis centrado en la arena de acción — participante y, por tanto, un poco más reflexivista—, en situaciones exógenas — basadas en las reglas principalmente y, por tanto, más positivista- o en los efectos de las interacciones - por lo que podría entrarse en el analiticismo-.

Por ello, y al centrar esta investigación en el papel de los actores, la ontología de la investigación será más analiticista, al buscar explicar la realidad, pero sin generar cambio social, sino brindar insumos para que en futuras investigaciones de análisis de políticas se tenga un conocimiento amplio sobre la problemática en el cambio de reglas en torno a las reservas forestales protectoras en Colombia, evidentes en el caso concreto con Río Blanco. Ahora bien, metodológicamente el modelo permite analizar ocho elementos clave que establecen las relaciones de los participantes (véase gráfica 1). 
La disputa por el territorio en el cambio en la Política de Ordenamiento Territorial...

Gráfica 1. Modelo del análisis del desarrollo institucional (ADI).

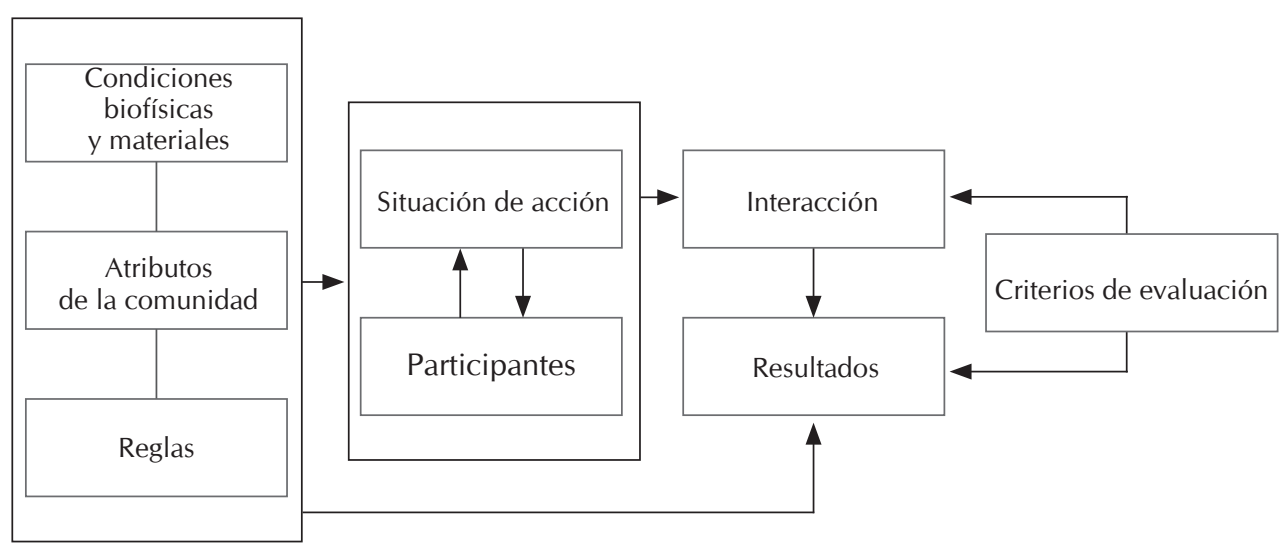

Fuentes: elaboración propia a partir de Ostrom (2015b, p. 53).

\subsubsection{Las reglas}

Las reglas son «prescripciones compartidas (debe, no debe o puede), comprendidas por todas las partes y que se hacen cumplir en forma predecible por los agentes responsables de controlar conductas e imponer sanciones». Las estrategias son «planes regularizados que los individuos hacen dentro de la estructura de incentivos producidos por las reglas, normas y expectativas del comportamiento probable de los demás en una situación afectada por condiciones físicas y materiales pertinentes» (Ostrom, 2000, p. 25). Los cambios institucionales se generan cuando la modificación de una regla afecta a los participantes y a las estrategias de las que disponen. Se afecta el control que tienen sobre los resultados y la información que poseen frente a los beneficios (Ostrom, 2015a, p. 247). El estudio del impacto en el cambio de la regla es lo que genera una nueva arena de acción.

Ostrom (2015a) establece tres tipos de reglas: las constitucionales, que determinan la situación de elección colectiva; las reglas de elección colectiva determinan las situaciones operativas; y las reglas operativas determinan las relaciones cotidianas de los actores con el entorno, esto es, las decisiones, acciones e información que se intercambian o retienen. Normalmente, el análisis de políticas públicas se centra en las reglas de elección colectiva. Al encontrarse en un nivel intermedio, los participantes poseen más autonomía y organización en la toma de decisiones e influyen en las reglas operativas, 
pudiendo establecer un cambio institucional (Ostrom, 2015b, p. 322). La regla constitucional es importante porque se centra en las normas generales que permean todo el ordenamiento jurídico. Pueden afectar todas las actividades y resultados operativos al establecer la regla de elección colectiva en un momento dado (Ostrom, 2015a, p. 111). La relación jerárquica existente entre los tres tipos de reglas permite a los participantes tener un grado de certidumbre en las estrategias que pueden adoptar para relacionarse con los RUC que tienen en su entorno. Hay un establecimiento normativo que les brinda seguridad jurídica en la toma de decisiones y permea el statu quo.

Para que el analista de política pueda establecer si se está ante una regla, Ostrom (2015b, p. 199) plantea la existencia de cinco componentes, denominados Adico: Atributo, Deóntico, objetlvo, Condiciones y De lo contrario. Estos son los encargados de establecer la influencia de la regla en una arena de acción determinada:

- Atributo: delimita a quién se le aplica el enunciado institucional o la posición específica que ocupa el participante.

- Deóntico: determina si la regla permite, obliga o prohíbe.

Objetivo: describe acciones o resultados de la situación de acción.

- Condiciones: determina cuándo y dónde las acciones o resultados están permitidos, obligados o prohibidos.

- O de lo contrario: las consecuencias dadas por no seguir la regla (Ostrom, 2015b, p. 198-199).

El componente objetivo -la I del Adico_ permite establecer las relaciones que se dan entre los participantes. Las conexiones están dadas por siete tipos de reglas: «posición, frontera, elección, agregación, información, pago y alcance» (Ostrom, 2015b, pp. 259-287). Las de posición se relacionan con el espacio que ocupa el participante en el proceso de toma de decisiones; las de frontera a la entrada y salida en las posiciones; las de elección se relacionan con las acciones de los participantes según su posición (pp. 261271); las reglas de agregación definen quién toma la decisión para generar el resultado final; las de información hablan sobre el estado de los participantes, la información que poseen y las acciones que realizan; las de pago son los beneficios o sanciones que se generan con la acción de los participantes; por último, las reglas de alcance se refieren a los resultados que se obtienen, los busquen o no (p. 272-281). 
La disputa por el territorio en el cambio en la Política de Ordenamiento Territorial...

\subsubsection{La arena de acción}

La arena de acción incluye a la situación de acción y a los participantes. El estudio de ambos permite «diagnosticar, explicar y predecir acciones y resultados» (Ostrom, 2000, p. 31). La situación de acción «está constituido por los participantes que toman decisiones dentro de una situación mediatizada por las características físicas, comunitarias e institucionales, y que darán como resultado diversos patrones de interacciones y resultados» (Ostrom y Hess, 2016, p. 79). Existen cuatro tipos de situación de acción, según la regla que la determine: las situaciones de acción operativas se basan en las acciones de los participantes que influyen directamente en el entorno; las situaciones de elección colectiva se basan en las acciones de los individuos que influyen en las reglas que determinan la situación operativa; las constitucionales intervienen en las reglas que determinan las situaciones de elección colectiva; por último, las meta constitucionales median en las reglas que determinan la situación constitucional (Ostrom, 2015b, p. 103).

Las relaciones verticales existentes entre las reglas y la situación de acción permiten entender la forma en que se da el cambio de nivel que genera un cambio institucional. Este cambio se da en tanto los participantes de una situación de acción utilizan las reglas de superior jerarquía para establecer la forma en que mejor se distribuyen sus derechos y deberes, y se mantenga o modifique el statu quo (Ostrom, 2015b). El establecimiento de una mejor situación implica la movilización de las estrategias y recursos que un individuo posee para convencer a otros que la regla propuesta para el cambio puede generar mejores condiciones de vida, considerando las restricciones actuales. Modificando la arena de acción en su totalidad, «es posible que el resultado de una modificación de niveles sea el mantenimiento de las reglas del statu quo» (p. 108).

El papel de los participantes es fundamental en la configuración de la situación de acción. Son quienes tienen la capacidad de influenciar en la utilización de estrategias que permitan establecer nuevas reglas. Utilizan el capital social, que «hace referencia al conjunto de redes de confianza interpersonal en las sociedades humanas, redes que pueden ser impulsadas por normas de reciprocidad y de ayuda mutua y que tienen una fuerte relación con las redes sociales y la participación en las asociaciones de la comunidad»» (Caballero, 2011, p. 31). Se crean redes de confianza que permiten construir acuerdos institucionales para entender y mejorar la apropiación que se 
realiza de los RUC (Ostrom, 2015a). Los intercambios de información llevan a procesos de aprendizaje incremental que modifican la situación de acción porque comprenden la forma en que un cambio en una regla afecta a todo el sistema. Esto permite reducir resultados inesperados que podrían contrariar lo que se espera conseguir (Ostrom, 2015b). Utilizando la comunicación como estrategia para alcanzar los objetivos personales o grupales de los participantes se aumenta el aprendizaje estratégico que permite «aprender sobre las situaciones de acción y el modo como los cambios en un componente de una situación influyen en las interacciones y los resultados» (p. 147). Con ello pueden predecir acciones de sus oponentes, entendiendo la situación de acción, mejorando o cambiando sus propias acciones, y elaborando reglas para obtener nuevos resultados.

\section{Metodología}

La investigación contó con un tipo de análisis cualitativo con un enfoque hermenéutico y de triangulación de información basada en el pattern matching (Trochim, 1989). La recopilación de información se realizó mediante entrevistas semiestructuradas, observación participante y la revisión documental de leyes, fallos judiciales, actos administrativos y el registro de

[178] archivos, referentes a la Reserva Forestal de Río Blanco y las áreas colindantes. Todos brindaron detalles verificables y contrastables entre sí.

El análisis mediante el pattern matching permitió que la información obtenida de las entrevistas analizadas a partir de la información relevante que evidenciara en tiempo, modo y lugar a los actores, y la utilización de sus estrategias frente a la modificación de las reglas se contrastara con la revisión documental documentada en una línea del tiempo mediante fichas de análisis y la observación participante. Se realizó un análisis inductivo a partir de los hallazgos propios de cada técnica de recolección para la concordancia por medio de la triangulación y para validar la hipótesis.

Se advierte que la descripción de los hallazgos y la información obtenida hace parte de archivos — principalmente demandas y actos administrativosdisponibles para todo el público, por ello, la mención expresa y personal de algunos actores responde a estos resultados. No hubo intención de realizar juicios de valor, ni de establecer algún tipo de responsabilidad penal o de otra índole, simplemente un análisis del deber ser en la actuación, conforme a los principios de primacía del interés general y de la naturaleza. 


\section{La aplicación del análisis de desarrollo institucional en el cambio del ordenamiento territorial en el terreno La Aurora, aledaño a Río Blanco}

Gráfica 2. Esquema del análisis de desarrollo institucional (ADI) en el cambio del ordenamiento territorial en La Aurora.

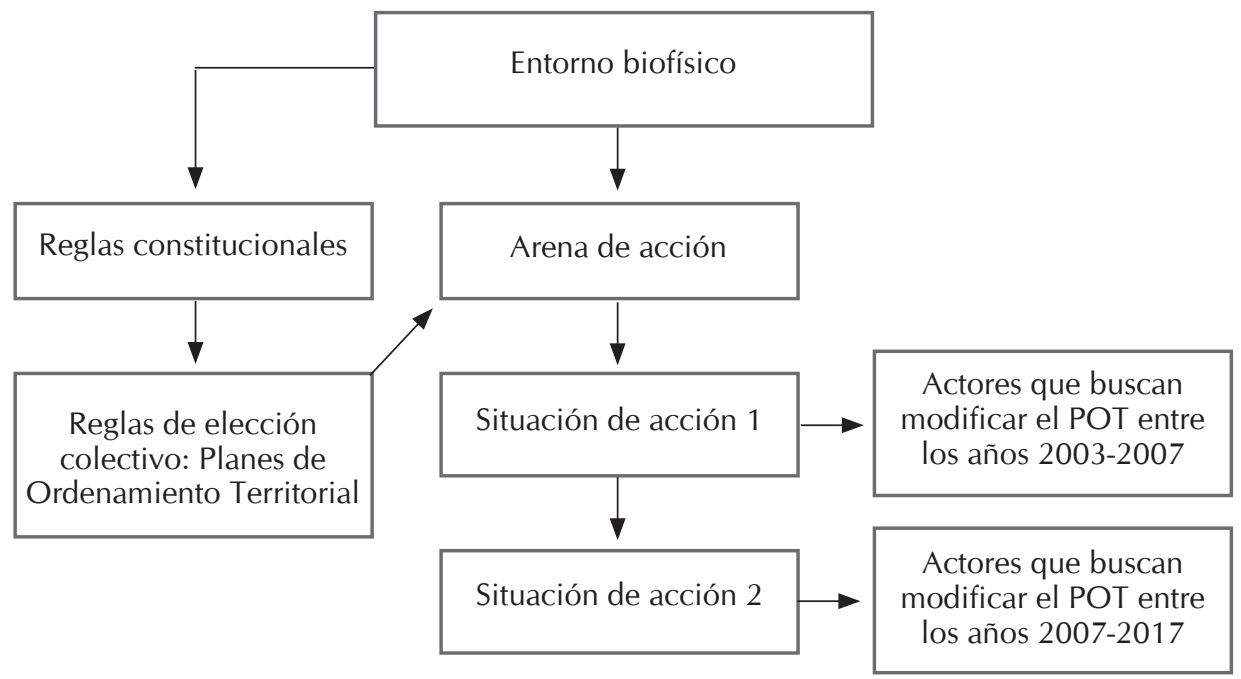

Fuentes: elaboración propia.

\subsection{El recurso de uso común: entorno biofísico}

Río Blanco es un bosque de niebla que presta el servicio ecosistémico de $35 \%$ del agua potable a la ciudad de Manizales (Corpocaldas, 2010). Posee una alta probabilidad de excluir a los beneficiarios del servicio de acueducto y de una sustracción alta porque el consumo del recurso hídrico por parte de una persona efectivamente disminuye la posibilidad de que otras puedan usarlo.

En 1990 las cuencas hidrográficas de Río Blanco y Quebrada Olivares se declararon área de reserva forestal protectora. Su conservación y manejo son obligación de las entidades municipales, responsabilidad delegada a la empresa prestadora del servicio de agua potable, Aguas de Manizales S. A E. S. P. Este RUC tiene la característica de ser administrado por el Estado, 
compitiendo con el derecho de propiedad privada del terreno colindante de La Aurora, pero con derechos de propiedad modificada en torno al terreno colindante que debería cumplir una función amortiguadora. En este caso interesa conocer las reglas de elección colectiva que se elaboraron para la conservación o para la modificación del suelo de La Aurora en pro de construir una urbanización. Dentro de los objetivos de conservación de la reserva se encuentra el restablecimiento de la conectividad con los bosques que están fuera de ella para mantener el corredor biológico que enlaza diversos municipios del país (Corpocaldas, 2010). Además, se considera que Río Blanco tiene gran diversidad ecosistémica, siendo un área prioritaria para la conservación de especies (Corpocaldas, Asocars y UNAL, 2013, p. 43).

El terreno de La Aurora es de propiedad privada y se encuentra en la cuenca de la quebrada Olivares, en la zona de bosque húmedo montano y bosque húmedo montano bajo, hace parte de las tierras que el plan de manejo de la reserva establece como áreas de conservación de suelos. Limita con Río Blanco al sur y al oeste:

- Sur. La vía carreteable que conduce a la Hacienda La Aurora. Distancia aproximada de 1.5 kilómetros.

[180] - Oeste. A 400 metros del predio del Sinaí a través del sendero que conduce a La Aurora, a través de la cota 2.250 en dirección Sur hasta encontrar el cruce de la vía de la hacienda la Aurora con la Quebrada Olivares (Corpocaldas, Asocars y UNAL, 2013, p. 39).

\subsection{Las reglas y la arena de acción}

Como se abordó en la discusión teórica, las reglas condicionan a la situación de acción y los participantes compiten por la modificación de una regla, vislumbrándose la imposibilidad de separar su análisis. De igual forma, está clara la relación vertical y simbiótica existente entre estos dos componentes del ADI.

La regla constitucional que guía todas las acciones de los participantes es la Ley 388 de 1997 que establece las reglas de elección colectiva. Estas son los POT que se formulan según los lineamientos de la ley mencionada. Las reglas operativas están marcadas por las relaciones cotidianas de los participantes, quienes encuentran diversos escenarios para oponerse al cambio en los POT, y en la formulación del Plan Parcial (PP) en el sector de La Aurora, así como 
La disputa por el territorio en el cambio en la Política de Ordenamiento Territorial...

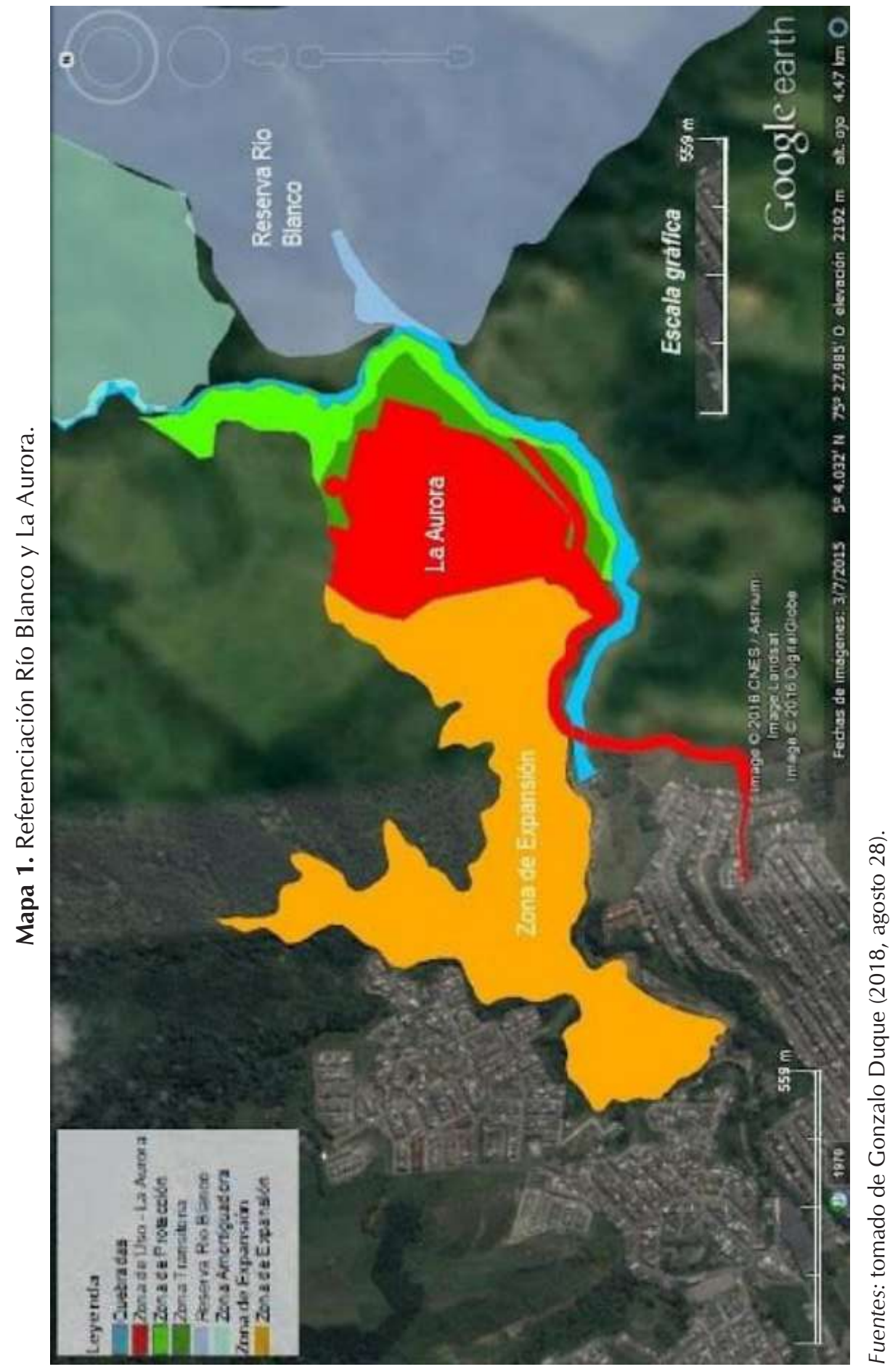


los permisos de construcción. Las estrategias de los participantes buscan el cambio de la regla de elección colectiva.

La regla constitucional establece que el ordenamiento del territorio se basa en la función pública del uso del suelo, el cuidado al medio ambiente y la protección del patrimonio cultural. La función social y ecológica de la propiedad y la prevalencia del interés general sobre el particular son parte de los principios básicos de esta ley (Ley 388, 1997, art. 2). Para la ordenación del territorio, los municipios deben establecer POT, los cuales están compuestos por tres partes: la general, que contempla las estrategias generales de largo y mediano plazo; la urbana, que contempla las políticas de mediano y corto plazo en el suelo urbano y de expansión urbana; y la rural, para garantizar la interacción entre los asentamientos urbanos y la cabecera municipal.

La parte general tiene un contenido estructural con una vigencia a largo plazo de doce años, que regula los temas ambientales relacionados con el uso, ocupación y aprovechamiento del suelo. También pueden hacer parte de las estrategias de mediano plazo del componente urbano. En cualquier caso, su modificación solo se puede hacer mediante la revisión general del POT o con motivos y estudios técnicos sustentados (Ley 388, 1997, art. 15).

En 2001 se estableció la primera regla de elección colectiva con el primer Plan de Ordenamiento Territorial de la ciudad de Manizales y, a su vez, la situación de acción donde los actores se confrontan para cambiar el statu quo que se implementará. En este se pretenden mantener estrategias de sostenibilidad ambiental que permitan su protección por medio de la promoción de una ocupación del suelo acorde con los recursos existentes y la función social de la propiedad, siguiendo los lineamientos de la regla constitucional que determina que los suelos de protección se establecen en las zonas con características geográficas, paisajísticas o ambientales, lugares en los cuales es imposible urbanizar por cuestiones ambientales. En el POT 2001 se cataloga a Río Blanco como un área de interés ambiental, así como la inexistencia de zonas de expansión urbana (ZEU).

Entre el 18 y 19 de marzo de 2003 se generaron deslizamientos de tierra que «ocasionaron 18 pérdidas de vidas humanas, 32 personas heridas, con el resultado adicional de 811 familias damnificadas, 74 viviendas destruidas y 104 quedaron afectadas. Fueron evacuadas 635 viviendas y 1700 quedaron en zonas de riesgo» (Alcaldía de Manizales, Corpocaldas y UNAL, s. f.). Con ello se declaró la emergencia que fue utilizada para justificar la 
La disputa por el territorio en el cambio en la Política de Ordenamiento Territorial...

necesidad de ampliar los terrenos para construcción, además de establecerse una proyección de crecimiento poblacional discutida en la propuesta de modificación del POT que contó con la presencia de la Cámara Colombiana de la Construcción (Camacol), la cual apoya la estrategia de ocupación del suelo de La Aurora (Acción Popular, cuaderno 1j, página 3662).

Los estudios presentados para adoptar el cambio en el POT no eran técnicos, simplemente evidenciaban lo que sucedía en el momento de los deslizamientos, los cuales no justificaban crear una ZEU en un terreno aledaño a una reserva forestal (Acuerdo 958 de 2017). Sin embargo, la presión de personas que actúan como egoístas racionales promovieron la creación del $\mathrm{PP}$, hicieron parte de la constructora tras previa ocupación de cargos públicos o influenciaban las acciones de la Alcaldía. Parece que se movilizó el aparato municipal en su beneficio.

Con la promulgación del Acuerdo 573 de 2003 se modificó el POT de 2001 con la consigna de Manizales como un municipio sin déficit de vivienda y así relocalizar los asentamientos ubicados en las zonas de alto riesgo o en laderas, y ampliando alternativas de vivienda digna. Se establece con ello que La Aurora es apta para vivienda de interés social. Por su parte, la Ley 4002 de 2004 establece que la revisión de las normas urbanísticas de carácter estructural que hacen parte de los contenidos ordinarios de los POT se realiza de forma excepcional si existen motivos que soporten la modificación. Mediante el Acuerdo Municipal 663 de 2007 se mantiene a La Aurora como ZEU, sosteniendo la modificación ya establecida en torno a la norma estructural contenida en el artículo 15 de la Ley 388. En esta ocasión se enfatiza en que la ausencia de proyectos urbanísticos estaba generando una nula promoción de viviendas nuevas.

Con la promulgación de La Aurora como ZEU se debió presentar una solicitud de sustracción de este terreno de la Reserva Forestal Central, de la cual hace parte Río Blanco. Esto se realizó en 2010, antes de que Jorge Alberto Vélez Jaramillo ${ }^{1}$ presentara ante la Alcaldía de Manizales la solicitud para iniciar con el PP, el cual fue concertado mediante el Decreto 289 de 2015; sin embargo, nunca se sustrajo de Río Blanco. El PP debía considerar como determinantes ambientales la sustracción de la reserva que se dio en el curso

\footnotetext{
${ }^{1}$ Propietario de una parte del terreno de La Aurora, promotor del cambio en el uso del suelo en el sector de La Aurora. En 2001 fue gerente de la empresa Aguas de Manizales y en 2012 miembro de la junta directiva de Corpocaldas, en representación de Camacol.
} 
de la solicitud y acreditar que se cumplía con la función amortiguadora de La Aurora frente a Río Blanco por encontrarse en el terreno colindante y aledaño — según el Plan de Manejo de la reserva, hacía parte fundamental para su protección—, como lo establece el Decreto 2372 de 2010. Debían existir estudios serios por parte de la entidad ambiental siguiendo los lineamientos de la Ley 99 de 1993 y no por parte del particular que establece medidas de mitigación del daño y las cataloga como función amortiguadora.

Una nueva situación de acción se generó cuando algunos ciudadanos que consideraban violentado su derecho a un ambiente sano, al equilibrio ecológico y al manejo y aprovechamiento racional de los recursos naturales interpusieron en 2012 una acción popular (Acción Popular, cuaderno 1c, página 95). Esta fue producto del aprendizaje estratégico evidenciado entre los habitantes de barrios aledaños a la zona a urbanizarse, quienes en conjunto con la Universidad de Caldas y la Personería de Manizales habían realizado recorridos en el entorno (comunicación personal, estudiante universitario, mayo 1.․․ 2019).

En las reuniones realizadas con la comunidad las entidades municipales concordaron en que existen otras fuentes abastecedoras de agua potable, que

[184] la reserva se encuentra a un kilómetro de la bocatoma más cercana y que los POT de 2003 y 2007 establecen que La Aurora es ZEU (Acción Popular, cuaderno 1c, página 174). Pese a ello, la Personería y algunas procuradurías judiciales para asuntos administrativos apoyaron la denuncia ciudadana para la protección de la reserva (Acción Popular, cuaderno 1g). Diversos actores individuales y colectivos en el transcurso de la acción popular comenzaron a participar en ella como coadyuvantes a nombre propio, de organizaciones sociales, ambientales, políticas, académicas, entre otros (Acción Popular, cuadernos 1 a 1k, 2, 3 y 4). Los argumentos para la protección de Río Blanco son de diversa índole, algunos se centran en explicar la violación a la norma constitucional, otros establecen condiciones ambientales especiales de Río Blanco y de La Aurora. Se alegan los efectos que tiene en el cambio climático, el daño a las especies endémicas y la importancia de los humedales para los ecosistemas. $^{2}$

Las entidades públicas que promovieron el cambio en el POT y que establecieron el PP coincidieron con la constructora en que las actuaciones

${ }^{2}$ Acción Popular, cuadernos 1 a 6, y Cabildo abierto realizado los días 5 y 6 de junio de 2017 en el concejo municipal de Manizales. 
La disputa por el territorio en el cambio en la Política de Ordenamiento Territorial...

realizadas se sujetaron a la ley y que cumplieron con los requisitos esperados (Acción Popular, cuaderno 1h). Por su parte, las acciones ciudadanas permiten entrever la existencia de un aprendizaje incremental y compartido de los diferentes contextos personales, con un interés común para idear estrategias más allá de las judiciales para mostrar a la ciudadanía la problemática que conllevó a la creación de la Coalición Todos Somos Río Blanco en 2017 (comunicación personal, estudiante universitario, mayo 1.․․ 2019).

Con la creación de la coalición se legitimaron las acciones separadas de los diversos actores sociales, permitiendo la visibilización de la problemática generada entre desarrollo territorial y protección ambiental. Al poseer una inclinación hacia la segunda, la coalición realizó marchas carnaval por toda la ciudad, con muestras artísticas y culturales que ponían en discusión el tema de las reservas y las fuentes de agua, la presentación de nuevas acciones judiciales y la realización de firmatones para que la ciudadanía las apoyara, así como la utilización de las redes sociales. ${ }^{3}$

La Alcaldía de Manizales (2017) radicó ante el Concejo Municipal un proyecto de revisión del POT. Se determinó que su socialización sería mediante un cabildo abierto a celebrarse el 5 y 6 de junio de 2017. De las ponencias presentadas, veinticuatro se referían a Río Blanco. Los participantes invocaron la premisa superior de la prevalencia del interés general sobre el particular, la función social y ecológica de la propiedad consagrado en el artículo 58 constitucional, y que, según la Corte Constitucional (Sentencia C-192 de 2016), las licencias de construcción no generan derechos adquiridos sobre el uso del suelo.

La inexistencia de estudios técnicos ajenos a la postura de la constructora sobre la afectación real a la reserva incita a aplicar el principio de precaución desarrollado por la Corte Constitucional, el cual indica que en los casos en que se desconozca con certeza el daño que una actividad, fenómeno o proceso puedan causar a la salud y al medio ambiente, las autoridades deben evaluar si ese riesgo es admisible o no, y con ello tomar la decisión final (Sentencia C-988 de 2004).

Debe tenerse en cuenta que es el almacenamiento y la provisión agua potable de la ciudad la que se discute, además del corte de los demás servicios

\footnotetext{
${ }^{3}$ Para conocer más acerca de las acciones realizadas por la coalición Todos Somos Río Blanco para la protección de la reserva, se puede ingresar a https://www.facebook.com/todossomosrioblanco/
} 
ecosistémicos de la reserva, como ser un corredor biológico o regulador el microclima de la ciudad. Debe existir una articulación entre el POT y los Objetivos de Desarrollo Sostenible (ODS) (ONU, 2012, junio 19) que permita coordinar acciones en el ámbito mundial para la protección del recurso hídrico y de los bienes naturales. Por último, se enfatiza en que el inicio del proyecto podría permitir más construcciones en el sector que afectarían de forma definitiva el corredor biológico, y el sostenimiento de la vida en la reserva si se tiene en cuenta el ciclo del agua y la necesidad de plantas especialmente diseñadas para la captación del recurso hídrico.

Todas las acciones y respuestas ciudadanas evidencian el conocimiento de la arena de acción y la previsión de las acciones que la constructora y los promotores del PP pudieron realizar. Con ello buscaban participar en los espacios donde el gobierno local actúa para modificar el statu quo que los POT de 2003 y de 2007 generaron en el terreno de La Aurora, esperando que este lugar se considere un territorio rural que protege de forma especial a Río Blanco. El resultado de las acciones ciudadanas se evidencia en que el Concejo Municipal modificó los POT de 2003 y 2007 en torno a La Aurora, siguiendo los lineamientos de la norma constitucional. Se determina que este lugar adquiere una categoría agropecuaria para la atenuación o reducción de las presiones sobre Río Blanco al promulgar el Acuerdo 958 de 2017. En este acuerdo se enfatiza que los análisis realizados para 2003 y 2007 no tenían una base sólida de argumentación, ni mucho menos datos bajo un análisis de condiciones reales para la toma de decisiones, por ello no se justificaba la determinación de ZEU en diversas partes de la ciudad (Acuerdo 958, 2017, p. 116).

\section{Conclusiones}

Según el artículo 15 de la Ley 388 de 1997, el POT debe ajustarse a las normas estructurales basadas en la protección y conservación ambiental. Su revisión solo se puede realizar cada doce años o por iniciativa del alcalde, si tiene estudios que la sustenten. En 2001 se estableció el primer POT en Manizales y la regla constitucional sirvió de referente para determinar que no era necesario instituir zonas de expansión urbana para la ciudad. En 2003, solo dos años después, se modificó el POT alegando el déficit de vivienda a causa de la declaratoria de desastre ese mismo año, producto de varios derrumbes que causaron daños en 178 hogares. Se invocó también una proyección de crecimiento poblacional. Con estos hechos se justificó el cambio en el uso del 
La disputa por el territorio en el cambio en la Política de Ordenamiento Territorial...

suelo del sector de La Aurora, pasando de zona rural a zona de expansión urbana (ZEU). Los documentos que se aportaron por parte de la Alcaldía Municipal no se eran técnicos, por tanto, el alcalde no tenía documentos de soporte que permitieran cambiar el uso del suelo según las exigencias de la norma constitucional. En 2007 una nueva modificación al POT de 2001 confirmó la decisión tomada en 2003, porque la gestión urbanística no se había desarrollado como debería, consecuencia de la poca gestión del suelo que contrastan con las cifras de aumento en la población, justificando la importancia de mantener a La Aurora como una ZEU para la promoción de la vivienda de interés social.

En estas dos revisiones se realizó una interpretación particular de los determinantes ambientales establecidos en la regla constitucional. Inicialmente, no habían pasado los doce años de la visión a largo plazo, además, los estudios presentados no eran técnicos, sino informes del alcalde y argumentos de daños a hogares como proyecciones de población que no motivaban, ni sustentaban la necesidad de modificar el contenido estructural del Plan de Ordenamiento Territorial de 2001.

En 2017 se adoptó la revisión ordinaria de los objetivos de largo plazo del POT, buscando la adecuación con la normativa internacional de protección a humedales. Al realizarse una verificación del crecimiento poblacional se observa que es casi nulo, por lo que las ZEU deben ser menores a las establecidas y no ubicarse en lugares que prestan servicios ambientales colectivos. De esta forma, La Aurora se convierte de nuevo en suelo rural que hace parte del corredor biológico de Río Blanco, territorio que necesita una protección adecuada en las zonas aledañas y circunvecinas.

Se cumple con ello la hipótesis planteada. La norma constitucional en la Ley 388 de 1997 determina que los Planes de Ordenamiento Territorial —norma de elección colectiva- deben cumplir con las normas estructurales ambientales, con ello los actores de la urbanización utilizaron sus intereses personales a favor de la modificación del terreno de La Aurora como ZEU cuando la necesidad de protección de Río Blanco exigía respetar la zona de amortiguación ante los posibles efectos de las acciones humanas sobre ella.

Tanto los ciudadanos que promotores de la expansión urbana en La Aurora como aquellos de la protección a Río Blanco coinciden en el respeto a la norma de elección constitucional, pero con una interpretación jurídica 
Tabla 1. Cambios en el Plan de Ordenamiento

Territorial de Manizales, 2003-2017.

\begin{tabular}{|c|c|c|}
\hline $\begin{array}{l}\text { Regla de elección } \\
\text { constitucional }\end{array}$ & Regla de elección colectiva & Consideraciones \\
\hline \multirow{4}{*}{$\begin{array}{l}\text { Ley } 388 \text { de } 1997 . \\
\text { Establece en su artículo } \\
15 \text { que el POT se } \\
\text { desarrolla mediante } \\
\text { normas urbanísticas, las } \\
\text { estructurales definen las } \\
\text { áreas de protección y } \\
\text { conservación ambiental. } \\
\text { Para su modificación se } \\
\text { debe hacer la revisión } \\
\text { general del POT (12 años) } \\
\text { o excepcionalmente, a } \\
\text { iniciativa del alcalde, si se } \\
\text { cuenta con estudios técnicos } \\
\text { motivados y sustentados. }\end{array}$} & $\begin{array}{l}\text { POT } 2001 . \\
\text { No existirán zonas de } \\
\text { expansión urbana en la } \\
\text { ciudad. El sector de La } \\
\text { Aurora hace parte del suelo } \\
\text { rural. }\end{array}$ & $\begin{array}{l}\text { Conforme a la Ley } 99 \text { de } 1993 \\
\text { y a la Ley } 165 \text { de } 1994 \text { La } \\
\text { Aurora cumple con funciones } \\
\text { ambientales de protección y } \\
\text { corredor biológico frente a Río } \\
\text { Blanco. }\end{array}$ \\
\hline & $\begin{array}{l}\text { POT } 2003 . \\
\text { El déficit de vivienda } \\
\text { producto de la declaratoria } \\
\text { de desastre de este año y } \\
\text { el crecimiento poblacional } \\
\text { hacen necesario crear ZEU, } \\
\text { entre ellas La Aurora. } \\
\end{array}$ & \multirow[t]{2}{*}{$\begin{array}{l}\text { No se ha cumplido el tiempo } \\
\text { para la revisión ordinaria y } \\
\text { según las consideraciones } \\
\text { del POT de } 2017 \text { no existían } \\
\text { documentos técnicos, ni } \\
\text { motivos suficientes que } \\
\text { justificaran el cambio en el } \\
\text { uso del suelo. }\end{array}$} \\
\hline & $\begin{array}{l}\text { POT } 2007 . \\
\text { La proyección de aumento } \\
\text { poblacional y los problemas } \\
\text { para la construcción de } \\
\text { vivienda en la ciudad hacen } \\
\text { necesario mantener las ZEU, } \\
\text { incluyendo a La Aurora. }\end{array}$ & \\
\hline & $\begin{array}{l}\text { POT } 2017 . \\
\text { Busca adecuarse la } \\
\text { normativa internacional de } \\
\text { protección ambiental. La } \\
\text { Aurora se ubica dentro de la } \\
\text { categoría rural. }\end{array}$ & $\begin{array}{l}\text { La Aurora se establece dentro } \\
\text { de la categoría agropecuaria } \\
\text { para la atenuación o } \\
\text { reducción de las presiones } \\
\text { sobre Río Blanco. }\end{array}$ \\
\hline
\end{tabular}

Fuentes: elaboración propia.

diferente. Los primeros encuentran en la norma razones para expandir la ciudad con base en el aumento poblacional y justifican así los cambios realizados en 2003 y 2007. Por su parte, quienes se oponen a la urbanización, han sido parte de un proceso de aprendizaje incremental que los llevó a ejercer una acción colectiva de vigilancia sobre las actuaciones del gobierno local y a exigir la modificación de las reglas en uso, esto permitió que en 2017 se estableciera una nueva regla de elección colectiva para la protección de Río Blanco. 
La disputa por el territorio en el cambio en la Política de Ordenamiento Territorial...

La hipótesis en términos de práctica se evidenciaría en la utilización de los determinantes ambientales bajo la necesidad de expansión territorial que contraponen intereses y visiones de desarrollo sostenible, bajo la idea de un POT que permita la utilización de zonas visibles como rurales para unos y como necesarias para la conservación ambiental para otros. Un grupo de ciudadanos se movilizó en función de unos valores de conservación y de una visión de largo plazo del territorio, en función de estos pretendieron y lograron establecer arreglos institucionales con las estrategias judiciales y de apertura de la problemática a un conjunto más amplio de ciudadanos, y generando presión sobre la Administración municipal y el Concejo de Manizales. Este último terminó adoptando la visión de la primacía del interés general sobre el particular y la necesidad de ordenar el territorio con relación a normas internacionales que permitan la conservación ambiental a largo plazo, lo que requirió de un nuevo POT en 2017.

\section{Referencias bibliográficas}

1. Alcaldía de Manizales (2017). Plan de Ordenamiento Territorial del Municipio de Manizales 2017-2031. Exposición de motivos. Proyecto de acuerdo «por el cual se adopta la revisión ordinaria de contenidos de largo plazo del Plan de Ordenamiento Territorial del Municipio de Manizales». Recuperado de ftp:// sig.manizales.gov.co/pot $\% 20$ manizales/POT\%20Acuerdo $\% 200958 \% 20 \mathrm{de} \% 20$ 2017/018_EXPOSICI\%C3\%93N\%20DE\%20MOTIVOS/EXPOSICI\%C3\%93N\%20 DE\%20MOTIVOS_POT_08-05-2017_V.1.6.pdf

2. Alcaldía de Manizales; Corporación Autónoma Regional deCaldas (Corpocaldas) y Universidad Nacional de Colombia. (s. f.). Gestión del riesgo Manizales 20012013. Recuperado de https://planeacion.manizales.gov.co/gestionriesgo/index.php/ memoria-221/45-gr-manizales-secciones/memoria-historica/102-2001-2013

3. Armenteras, Dolors; Cadena, Camilo y Moreno, Rocío del Pilar. (2007). Evaluación de estado de los bosques de niebla y de la meta 2010 en Colombia. Bogotá D. C.: Instituto de Investigación de Recursos Biológicos Alexander Von Humboldt. Recuperado de http://repository.humboldt.org.co/bitstream/ handle/20.500.11761/31397/126.pdf? sequence $=1$

4. Bowles, Samuel. (2004). Microeconomics, Behavior, Institutions, and Evolution. New York: Russell Sage Foundation.

5. Caballero, Gonzalo. (2011). Economía de las instituciones: de Coase y North a Williamson y Ostrom. Ekonomiaz, 77 (2), pp. 14-44.

6. Caldas. Tribunal Administrativo. Acción Popular 17001233300020120013700 (2012). Manizales. pp. 1-3835. 
7. Colombia. Alcaldía de Manizales. Decreto 289. (28 de mayo de 2015). Por el cual se adopta el plan parcial en suelo de expansión urbana sector La Aurora. Gaceta Municipal. Manizales, 2015. N. ${ }^{\circ} 098$.

8. Colombia. Concejo de Manizales. Acuerdo 508. (12 de octubre de 2001). Por medio del cual se adopta el Plan de Ordenamiento Territorial del municipio de Manizales. Recuperado de http://www.curaduriamanizales.com/docs/ AcuerdoNo.508Oct.122001.pdf

9. Colombia. Concejo de Manizales. Acuerdo 573. (24 de diciembre de 2003). Por medio del cual se modifica el Plan de Ordenamiento Territorial del municipio de Manizales. Recuperado de http://www.curaduriamanizales.com/docs/ AcuerdoNo.573Dic.242003.pdf

10. Colombia. Concejo de Manizales. Acuerdo 663. (13 de septiembre de 2007). Por medio del cual se adopta la revisión del plan de Ordenamiento Territorial del municipio de Manizales. Recuperado de ftp://sig.manizales.gov.co/pot\%20manizales/ Historicos/Acuerdo\%20663-2007/Acuerdo_663_sep_13_\%202007.pdf

11. Colombia. Concejo de Manizales. Acuerdo 958. (2 de agosto de 2017). Por el cual se adopta la revisión ordinaria de contenidos de largo plazo del Plan de Ordenamiento Territorial del municipio de Manizales. Recuperado de ftp:// sig.manizales.gov.co/pot \% 20manizales/POT\%20Acuerdo \% 200958\% 20de \% 20 2017/006_ACUERDO/ACDO.\% 200958-02-08-2017\% 20POR\% 20EL \% 20

[190] CUAL\%20SE\%20ADOPTA\%20LA\%20REVISION\%20ORDINARIA\%20DE\% 20 CONTENIDOS\%20DE\%20LARGO\%20PLAZO\%20DEL\%20POT.pdf

12. Colombia. Congreso de la República. Ley 99. (22, diciembre, 1993). Por la cual se crea el Ministerio del Medio Ambiente, se reordena el Sector Público encargado de la gestión y conservación del medio ambiente y los recursos naturales renovables, se organiza el Sistema Nacional Ambiental, SINA, y se dictan otras disposiciones. Diario Oficial. Bogotá, D. C., 1993. N. ${ }^{\circ} 41146$.

13. Colombia. Congreso de la República. Ley 388. (18 de julio de 1997). Por la cual se modifica la Ley $9^{a}$ de 1989, y la Ley $3^{\text {a }}$ de 1991 y se dictan otras disposiciones. Diario Oficial. Bogotá, D.C., 1997. N. ${ }^{\circ} 43127$

14. Colombia. Corte Constitucional. Sentencia C-339. (7 de mayo de 2002). Recuperado de https://www.corteconstitucional.gov.co/RELATORIA/2002/C-339-02. htm

15. Colombia. Corte Constitucional. Sentencia C-988. (12 de octubre de 2004). Recuperado de https://www.corteconstitucional.gov.co/relatoria/2004/c-988-04.htm

16. Colombia. Corte Constitucional. Sentencia C-192. (20 de abril de 2016). Recuperado de https://www.corteconstitucional.gov.co/RELATORIA/2016/C-192-16. htm 
La disputa por el territorio en el cambio en la Política de Ordenamiento Territorial...

17. Colombia. Ministerio de Ambiente, Vivienda y Desarrollo Territorial. Decreto 2372. (1. ${ }^{\circ}$ de julio de 2010). Por el cual se reglamenta el Decreto Ley 2811 de 1974, la Ley 99 de 1993, la Ley 165 de 1994 y el Decreto Ley 216 de 2003, en relación con el Sistema Nacional de Áreas Protegidas, las categorías de manejo que lo conforman y se dictan otras disposiciones. Diario Oficial. Bogotá, D.C., 2010. N. ${ }^{4} 45757$.

18. Corporación Autónoma Regional de Caldas (Corpocaldas). (2010). Reserva forestal protectora de las cuencas hidrográficas de Río Blanco y quebrada Olivares: plan de manejo. Manizales: Corpocaldas. Recuperado de http://www.corpocaldas. gov.co/publicaciones/576/Plan_Manejo_.pdf

19. Corporación Autónoma Regional de Caldas (Corpocaldas); Asociación de Corporaciones Autónomas Regionales (Asocars) y Universidad Nacional sede Manizales. (2013). Plan de ordenación y manejo cuenca hidrográfica del río Chinchiná. Fase de Zonificación ambiental. Corpocaldas. Recuperado de http://www. corpocaldas.gov.co/publicaciones/1508/2017/03-09/03-FaseZonificacionAmbiental. pdf

20. Duque Escobar, Gonzalo. (2018, agosto 28). Río Blanco, en Manizales, está amenazado por un conflicto de ocupación territorial. La Silla Vacía. Recuperado de https://lasillavacia.com/silla-Ilena/red-paisa/historia/rio-blanco-en-manizales-estaamenazado-por-un-conflicto-de-ocupacion

21. Estenssoro, Fernando. (2015). El ecodesarrollo como concepto precursor del desarrollo sustentable y su influencia en América Latina. Universum, 30 (1), pp. 8199. https://doi.org/10.4067/S0718-23762015000100006

22. Hardin, Garrett. (1968). The Tragedy of the Commons. Science, 162 (3859), pp. 1243-1248. https://doi.org/10.1126/science.162.3859.1243

23. Hess, Charotte y Ostrom, Elinor. (2016). Los bienes comunes del conocimiento. Madrid: Traficantes de Sueños, Instituto de Altos Estudios Nacionales.

24. Massiris, Ángel. (2002). Ordenación del territorio en América Latina. Scripta Nova, 6 (125). Recuperado de http://www.ub.edu/geocrit/sn/sn-125.htm

25. Organización de las Naciones Unidas (ONU). (2012, junio 19). Conferencia de las Naciones Unidas sobre el Desarrollo Sostenible. Tema 10. Documento final de la Conferencia. Recuperado de https://rio20.un.org/sites/rio20.un.org/files/aconf.216-I-1_spanish.pdf.pdf

26. Ostrom, Elinor. (2000). Elección racional institucional: Evaluación del marco de análisis y desarrollo institucional. En: Paul Sabatier (ed.). Teorías del proceso de las políticas públicas (pp. 23-68). Buenos Aires: Proyecto de Modernización del Estado.

27. Ostrom, Elinor (ed.). (2015a). El gobierno de los comunes: la evolución de las instituciones de acción colectiva. México, D. F.: Fondo de Cultura Económica.

28. Ostrom, Elinor. (2015b). Comprender la diversidad institucional. México, D. F.: Fondo de Cultura Económica, Universidad Autónoma Metropolitana. 
29. Roth, André-Noel. (2010). Las políticas públicas y sus principales enfoques analíticos. En: Roth, André-Noel (ed.). Enfoques para el análisis de políticas públicas (pp. 17-56). Bogotá, D. C.: Universidad Nacional de Colombia.

30. Roth, André-Noel. (2018). Políticas públicas: formulación, implementación y evaluación. Bogotá, D. C.: Aurora.

31. Trochim, William. (1989). Outcome Pattern Matching and Program Theory. Evaluation and Program Planning, 12, pp. 355-366. https://doi.org/10.1016/01497189(89)90052-9

32. Zurbriggen, Cristina. (2011). Gobernanza: una mirada desde América Latina. Perfiles Latioamericanos, 38, pp. 39-64. 